\title{
Should patients with cystic fibrosis infected with Burkholderia cepacia undergo lung transplantation?
}

\author{
A K Webb, J Egan
}

A medical issue currently causing much discussion in cystic fibrosis centres and transplant units is the concern that patients with cystic fibrosis colonised with Burkholderia cepacia may have a poor outcome following transplantation. ${ }^{12}$ As the supply of donor organs continues to decline, the question arises as to whether these patients should be excluded from transplantation. Is there sufficient clinical and scientific information to reach a decision to exclude this group of patients from lung transplantation, which offers increased survival? Are there any solutions? Is such a decision ethically correct? Many issues need to be carefully addressed before such a finite conclusion is reached.

Burkholderia cepacia, previously recognised as a plant pathogen (Latin cepia= onion), emerged in the early 1980s as a human pathogen. It has a particular predisposition to infect patients with cystic fibrosis. Infection with $B$ cepacia is distinguished from infection with other major cystic fibrosis pathogens such as Pseudomonas aeruginosa by accelerated lung disease in some patients with mild to moderate lung disease. ${ }^{3-6}$ Overwhelming septicaemia and necrotising pneumonia due to $B$ cepacia are well described, ${ }^{5-7}$ events which are extremely rare with $P$ aeruginosa infection. The precise reasons for the increased virulence of $B$ cepacia have been investigated but not identified, ${ }^{8}$ but many isolates exhibit intrinsic multiple resistance to all available antibiotics. ${ }^{9-11}$

It is important to know the current number of paediatric and adult patients infected with $B$ cepacia in the UK, Europe, and North America. Pitt et al recently identified 178 patients with cystic fibrosis in 17 paediatric and adult cystic fibrosis centres in the UK who harboured $B$ cepacia. ${ }^{11}$ The United States Cystic Fibrosis Foundation registry report published in 1994 identified 538 patients (3.2\%) growing $B$ cepacia. A current epidemiological survey has identified $221(6 \%) B$ cepacia positive patients in the UK and 333 (5\%) B cepacia positive patients in Europe. Worldwide there is therefore a small but significant number of patients infected with $B$ cepacia who potentially will need a transplant.

Although the numbers of patients with cystic fibrosis infected with $B$ cepacia are relatively small, the current prevalence is likely to persist. Epidemiological studies using bacteriocin typing, multilocus enzyme electrophoresis, PCR ribotyping, and pulsed-field gel electrophoresis have shown that $B$ cepacia may be highly transmissible between patients. ${ }^{12-15}$ Cross infection occurs usually by direct person-to-person spread; a third party source is uncommon. Some strains may be more transmissible than others. An epidemic strain is described with novel cable pili which bind to cystic fibrosis mucin and epithelial cell receptors. ${ }^{1516}$ The incidence of $B$ cepacia is now declining as a consequence of strict segregation policies in cystic fibrosis centres in North America and the UK. ${ }^{1317}$ Although some centres have described the end of their epidemic, ${ }^{6}$ segregation strategies have not completely eliminated acquisition because transmission still occurs between $B$ cepacia positive and negative patients who congregate socially. ${ }^{18}$ Outbreaks are also described in cystic fibrosis centres which have not yet adopted a segregation policy. ${ }^{19}$

Demand for donor organs currently exceeds a decreasing supply and a third of all patients with cystic fibrosis listed for transplantation die on the waiting list. ${ }^{20}$ Death rates from road traffic accidents and intracranial haemorrhage have decreased because of active strategies to resolve these problems. The donor pool may also be reduced in countries with limited availability of intensive care beds. Great variability also exists between regions in the UK for rates of lung donation - for example, in 1994 there were 7.1 cadaveric thoracic organ donations per million population (PMP) made in the North West of England compared with 9.8 PMP for the Northern/Yorkshire region which suggests that local factors may influence donor rates.

New approaches have been advocated to improve organ donation. Continuing education to medical and nursing staff on the beneficial aspects of transplantation is crucial to increase limited organ donation. In Spain there has been a $40 \%$ increase in the rate of organ donation on the basis of constructing a widespread coordination network which focuses on donor detection rather than only concentrating on retrieval. Emphasis on skilled family communication and the use of donor management protocols at an early stage have been advocated. The limited supply of organ donation has resulted in Starnes et al pioneering lung donation from living relatives to patients. ${ }^{21}$ This strategy may be helpful for patients with cystic fibrosis who are young and who have youthful relatives as potential donors.

What is the prognosis of $B$ cepacia infected patients following transplantation? The evidence is conflicting. Reports from a North American centre have described a mortality rate of $75 \%$ following transplantation in 15 patients with cystic fibrosis infected with $B$ cepacia. ${ }^{12}$ However, in $50 \%$ of those patients infected with $B$ cepacia the organism was identified for the first time after transplantation. This implies either the organism was not identified by appropriate microbiological techniques prior to transplantation or the organism was acquired following transplant surgery. Following transplantation patients are vulnerable to nosocomial infections. Spread of $B$ cepacia in non-cystic fibrosis immunocompromised patients is well recognised. ${ }^{22}$ In contrast, two subsequent reports - one from North America and one from the UK - found no excess mortality following transplantation in those patients infected with $B$ cepacia. ${ }^{23}{ }^{24}$ In a report from the UK the combined data from three separate transplant programmes did not find a greater mortality in 14 patients colonised with $B$ cepacia. In a report from North Carolina $12 B$ cepacia positive patients had a similar outcome to other transplanted patients. ${ }^{23}$

It is appropriate at this point to note that organisms other than $B$ cepacia may potentially affect clinical outcome following transplantation. These organisms can include multiple resistant pseudomonads, methicillin resistant staphylococcus (MRSA), Aspergillus fumigatus, Xanthomonas maltophilia, and $B$ gladioli. Two recent studies have shown that the presence of these resistant organisms in patients with cystic fibrosis before transplantation does not predict a worse outcome following transplantation than 
for patients infected with sensitive organisms. ${ }^{2526}$ It is crucial that these patients are in a good nutritional state and a stable clinical state when transplanted.

Overall, patients with cystic fibrosis infected with $B$ cepacia are probably at greater risk of postoperative complications than $P$ aeruginosa infected patients. Following transplant surgery, severe infective episodes have been reported which include septicaemia, pericarditis, pneumonia, and empyemas. ${ }^{627}$ However, although some patients infected with $B$ cepacia may have a difficult postoperative course, prolonged survival in a reasonable number of carefully selected patients does occur when cumulative evidence is examined from different centres. ${ }^{2324}$ Removing the lungs, the primary focus of $B$ cepacia infection, is the purpose of transplant surgery and in some patients $B$ cepacia infection has been eradicated following transplantation, although recolonisation commonly occurs from the upper airways.

In the presence of conflicting reports can we identify those $B$ cepacia positive patients who might do badly? This may depend upon the virulence of the organism and the host responses to this unusual opportunistic pathogen. However, very little is understood about the host-pathogen interaction which can identify "at risk" patients. Prognostic statements on the clinical consequences of colonisation are difficult; even in epidemic outbreaks when patients are colonised by the same strain, some patients may remain asymptomatic whilst others succumb to rapid fatal decline. ${ }^{613}$

Members of the species presently identified as $B$ cepacia are heterogeneous both in their genomic and phenotypic profile. ${ }^{28-30}$ One study identified more than 50 ribotype patterns in 178 patients. ${ }^{11}$ In the same study, isolates of an epidemic strain with the same genomic profile showed a very variable antibiotic susceptibility. In our unit six patients have eliminated the organism from their sputum for more than three years and another five are colonised intermittently. Other units have noted similar findings. ${ }^{6}$

Identifying differing host responses in those patients infected with $B$ cepacia may be just as important as the virulence of the organism. A UK multicentre study has shown that the exaggerated host response, characteristic of cystic fibrosis, is greatest in the presence of infection with $B$ cepacia; levels of the inflammatory markers Creactive protein and neutrophil elastase $\alpha_{1}$-antiproteinase complex were significantly higher during $B$ cepacia associated exacerbations than in exacerbations due to $P$ aeruginosa alone. ${ }^{31}$ The lipopolysaccharide of $B$ cepacia can induce pro-inflammatory cytokines including tumour necrosis factor to a level 10 times greater than that induced by $P$ aeruginosa. ${ }^{32}$

Despite the general observation that lung disease may be accelerated by infection with $B$ cepacia, patients with cystic fibrosis do respond to maximum multidisciplinary medical care and, in particular, to intravenous antibiotics. Peckham et al showed that, despite poorer lung function and greater in vitro antibiotic resistance, $B$ cepacia positive patients had as good a response as $B$ cepacia negative patients to intravenous antibiotics using lung function, body weight, and inflammatory markers as clinical end points. ${ }^{33}$ Aggressive antibiotic therapy can reduce inflammatory markers to pre-exacerbation levels. ${ }^{31}$

What can be done to decrease the greater risk for $B$ cepacia positive patients at time of transplantation? Some patients infected with $B$ cepacia have such rapid disease progression and are so clinically unstable with fevers and constant infection that they are unsuitable for transplantation. Following transplantation such patients are likely to develop uncontrollable sepsis in the presence of a high level of immunosuppression. However, other patients with $B$ cepacia infection have a slower disease progression, are well nourished, clinically stable, and their pulmonary infection is responsive to antibiotics.

There needs to be greater knowledge of what determines bacterial virulence and exaggerated host responses for the individual patient infected with $B$ cepacia. Perhaps $B$ cepacia positive patients should be listed earlier for transplantation. Patients infected with $P$ aeruginosa have a slower clinical progression and, at time of listing, can expect to wait up to two years for a transplant. $B$ cepacia positive patients may need listing earlier for transplantation with better spirometric values, nutrition, and a more stable disease state than the standard criteria used when listing a cystic fibrosis patient. Patients with cystic fibrosis may be reluctant to consider this option if their quality of life is reasonable.

Suggested measures to reduce the postoperative risk for $B$ cepacia positive patients might include preoperative and postoperative sinus drainage, modifying immunosuppressive protocols, and commencing aggressive intravenous antibiotic prophylaxis at the time of transplantation based upon knowledge of clinical response prior to surgery irrespective of antibiotic resistance. ${ }^{2}$

Is it ethical to exclude from transplantation a significant number of patients with cystic fibrosis infected with $B$ cepacia? To deny them the opportunity would be to diminish any hope of a future. Are risk factors, limited organ supply, potential postoperative problems, and health economics good enough reasons to make a decision of exclusion? Should there exist an aristocracy of the more fortunate? It can be argued on ethical grounds that health care (in this case, transplantation) requires that each patient with cystic fibrosis, irrespective of infecting organism or risk, is entitled to the opportunity of having a transplant as long as there is a chance of benefit. ${ }^{34}$ Against this precept is the decreasing supply of donor organs and the most effective way of using this limited resource.

These are difficult issues, but until there is unequivocal evidence of an absence of benefit for patients with $B$ cepacia who undergo transplantation, an equitable approach ought to be applied - that is, equal access for equal needs.

Above all, the emphasis should remain on improving donor availability rather than excluding patients from transplantation.

We are indebted to Drs A Woodcock, M Dodd, D Stableforth, J Harris, and J Govan for their robust comments in the preparation of this manuscript.

Correspondence: Dr AK Webb.

Bradbury CF Unit,

North West Lung Centre,

Wythenshawe Hospital,

Southmoor Road,

Manchester M23 9LT, UK

A K WEBB

J EGAN

1 Snell GI, de Hoyos A, Krajden M, Winton T, Maurer JR. Pseudomonas cepacia in lung transplant recipients with cystic fibrosis. Chest 1993;103: 466-71

2 Ramirez JC, Patterson GA, Winton TL, De Hoyos AL, Miller JD, Maure JR. Bilateral lung transplantation for cystic fibrosis. F Thorac Cardiovasc Surg 1992;103:287-94.

3 Thomassen MJ, Demko CA, Klinger JD, Stern RC. Pseudomonas cepacia colonisation amongst patients with cystic fibrosis. A new opportunist. Am Rev Respir Dis 1985;131:791-6.

4 Tablan O, et al. Pseudomonas cepacia colonisation in patients with cystic fibrosis: risk factors and clinical outcome. F Pediatr 1985;107:382-7.

5 Isle A, Macluskey A, Corey M, Gould R, Prober C, Fleming P, et al. Pseudomonas cepacia infection in cystic fibrosis: an emerging problem. $\mathcal{F}$ Peudomonas cepacia infectio

Mediatr 1984;104:206-10. DL, et al. Outcome for patients colonised with Burkholderia cepacia in a Birmingham adult cystic fibrosis clinic and the end of an epidemic. Thorax 1996;51:374-7

7 Glass S, Govan JRW. Pseudomonas cepacia - fatal pulmonary infection in a patient with cystic fibrosis. F Infect 1986;13:157-8

8 Nelson JW, Butler SL, Krieg D, Govan JR. Virulence factors of Burkholderia cepacia. FEMS Immunol Med Microbiol 1994;8:89-98.

9 Bhakta DR, Leader I, Jacobson R, Robinson-Dunn B, Honicky RE, Kumar A. Antibacterial properties of investigational, new, and commonly used 
antibiotics against isolates of Pseudomonas cepacia in Michigan. Chemotherapy 1992;38:319-23.

10 Lewin C, Doherty C, Govan JRW. In vitro activities of meropenem, PD 127391 and PD 131628, ceftazidime, chloramphenicol, co-trimoxazole and ciprofloxacin against Pseudomonas cepacia. Antimicrob Agents Chemother 1993;37:123-5.

11 Pitt TL, Kaufman ME, Patel PS, Benge CA, Gaskin S, Livermore DM. Type characterisation and antibiotic susceptibility of Burkholderia (Pseudomonas) isolates from patients with cystic fibrosis in the United Kingdom and the Republic of Ireland. F Med Microbiol 1996;44:203-10.

12 LiPuma JJ, Dasen SE, Neilson DW, Stern RC, Stull TL. Person-to-person transmission of Pseudomonas cepacia between patients with cystic fibrosis. Lancet 1990;336:1094-6.

13 Govan JR, Brown PH, Maddison J, Doherty CJ, Nelson JW, Dodd M, et al. Evidence for transmission of Pseudomonas cepacia by social contact in cystic fibrosis. Lancet 1993;342:15-19.

14 Smith DL, Gumery LB, Smith EG, Stableforth DE, Kauffmann ME, Pitt TL. Epidemic of Pseudomonas cepacia in an adult cystic fibrosis unit: evidence of person-to-person transmission. F Clin Microbiol 1993;31: 3017-22.

15 Sun L, Jiang RZ, Steinbach S, Holmes A, Campanelli C, Forstner J, et al. The emergence of a highly transmissable lineage of $\mathrm{cbl}(+)$ Pseudomonas (Burkholderia) cepacia causing CF centre epidemics in North America and (Burkholderia) cepacia causing CF cent

16 Sylvester FA, Sajjan US, Forstner JF. Burkholderia (Basonym Pseudomonas) cepacia binding to lipid receptors. Infect Immunol 1996;64:1420-5.

17 Thomassen MJ, Demko CA, Doershuk CF, Stern RC, Klinger JD. Pseudomonas cepacia: decrease in colonisation in cystic fibrosis patients. Am Rev Respir Dis 1986;134:669-71.

18 Smith DL, Smith EG, Gumery LB, Stableforth DE. Pseudomonas cepacia infection in cystic fibrosis. Lancet 1992;339:252.

19 Whiteford ML, Wilkinson JD, McColl JH, Conlon FM, Michie JR, Evans TJ, et al. Outcome of Burkholderia (Pseudomonas) cepacia colonisation in children with cystic fibrosis following a hospital outbreak. Thorax 1995; 50:1194-8.

20 Ryan PJ, Stableforth DE. Referral for lung transplantation: experience of a Birmingham Adult Cystic Fibrosis Centre between 1987 and 1994. Thorax 1996;51:302-5.
21 Starnes VA, Barr ML, Cohen RG, Schenkel FA. Living related lung transplantation in cystic fibrosis. Pediatr Pulmonol 1994;10(Suppl): 128-9.

22 Yamagishi Y, Fujita K, Takigawa K, Negayama T, Nakazawa T, Takahar J. Clinical features of Pseudomonas cepacia pneumonia in an epidemic among immunocompromised patients. Chest 1993;103:1706-9.

23 Flume PA, Egan TM, Paradowiski LJ, Detterbeck FC, Thomson JT, Yankaskas JR. Infectious complications of lung transplantation; impact of cystic fibrosis. Am $\mathcal{F}$ Respir Crit Care Med 1994;149:1601-7.

24 Egan JJ, McNeil K, Bookless B, Gould K, Corris P, Higgenbottam T, et al. Post-transplantation survival of cystic fibrosis patients infected with Pseudomonas cepacia. Lancet 1994;334:552-3.

25 Gabbay E, Archer L, Nkere U, Wrightson N, Gould K, Dark JH, et al. Results of pulmonary transplantation in patients with cystic fibrosis colonised by multiply resistant pseudomonads. Pediatr Pulmonol 1996 13(Suppl):311.

26 Kanj SS, Tapson V, Madden J, Davis D, Browning I. Pre-transplant resistant organisms do not predict outcome for lung transplantation. Pediatr Pulmonol 1996;13(Suppl):311.

27 Noyes BE, Michaels MG, Kurland G, Armitage JM, Orenstein DM. Pseudomonas cepacia empyema necessitans after lung transplantation in two patients with cystic fibrosis. Chest 1994;105:1888-91.

28 Butler SL, Doherty CJ, Hughes JE, Nelson JW, Govan JRW. Burkholderia cepacia and cystic fibrosis: do natural environments present a potential hazard? $\mathcal{F}$ Clin Microbiol 1995;33:1001-4.

29 Simpson IN, Finlay J, Winstanley DJ, et al. Multiresistant isolates possessing characteristics of both Burkholderia (Pseudomonas) cepacia and Burkholderia characteristics of both Burkholderia (Pseudomonas) cepacia and Burkholderia
gladioli from patients with cystic fibrosis. 7 Antimicrob Chemother 1995;34: gladioli 1 . 253 . 61 .

30 Govan JRW, Hughes JE, Vandammme P. Burholderia cepacia: medical taxonomic and ecological issues. F Med Microbiol 1996;45:395-407.

31 Elborn JS, Dodd M, Maddison J, et al. Clinical and inflammatory responses in CF patients infected with Pseudomonas a Shepacia. Pediatr Pulmonol 19RW.

32 Shaw D, Poxton IR, Govan JRW. Biological activity of Burkholderia (PseudoTIm Imunol Med Microbiol 1995 ,

33 Peckham D, Crouch S, Humphreys H, Lobo B, Tse A, Knox AJ. Effect of antibiotic treatment on inflammatory markers and lung function in cystic fibrosis patients with Pseudomonas cepacia. Thorax 1994;49:803-7.
4 Harris J. What is the good of health care? Bioethics 1996;4:269-91. 